\title{
Hysteretic metal-ferroelectric- semiconductor capacitors based on PZT/ZnO heterostructures
}

\author{
E Cagin ${ }^{1}$, D Y Chen ${ }^{2}$, J J Siddiqui ${ }^{3}$ and J D Phillips \\ Solid State Electronics Laboratory, Electrical Engineering and Computer Science \\ Department, University of Michigan, Ann Arbor, MI 48109, USA \\ E-mail: cagin@eecs.umich.edu
}

Received 1 December 2006, in final form 14 February 2007

Published 4 April 2007

Online at stacks.iop.org/JPhysD/40/2430

\begin{abstract}
Interfacing of ferroelectric and semiconductor materials provides a means of coupling unique properties associated with ferroelectric materials to high performance semiconductor devices. In this work we report the electronic properties of ferroelectric/ZnO heterostructures, where $(\mathrm{Pb}, \mathrm{Zr}) \mathrm{TiO}_{3}(\mathrm{PZT})$ is used as a prototypical ferroelectric oxide. Metal-PZT-metal structures demonstrate ferroelectric hysteresis with remanent polarization of $28 \mu \mathrm{C} \mathrm{cm}^{-2}$ and coercive field of $75 \mathrm{kV} \mathrm{cm}^{-1}$ for a loop of $15 \mathrm{~V}$. The metal-PZT-ZnO capacitor structures demonstrate a characteristic metal-insulator-semiconductor capacitance-voltage $(C-V)$ behaviour with a hysteretic memory window of approximately $4 \mathrm{~V}$. The heterostructure $C-V$ characteristics do not change significantly with varying frequency. Metal-PZT-ZnO capacitors are also used as part of a simple RLC circuit to demonstrate the ability to shift resonant frequency of the circuit with switching ferroelectric polarization.
\end{abstract}

\section{Introduction}

Ferroelectric-semiconductor heterostructures are of great interest for future devices where the unique properties of such materials can be used for electronic and optoelectronic applications. Perovskite oxides with switchable polarization, electrooptic, pyroelectric, piezoelectric properties and voltage tunable dielectric constants are highly desirable for sensors and memory devices. Interfacing ferroelectric oxides with semiconductors will allow us to take advantage of such materials properties for multi-functional devices. Ferroelectric oxides deposited on semiconductors such as $\mathrm{Si}$ [1] and $\mathrm{AlGaN} / \mathrm{GaN}$ [2] have been studied previously.

The interface quality of ferroelectric/semiconductor heterostructures is of utmost importance for effectively coupling

\footnotetext{
1 Author to whom any correspondence should be addressed.

${ }^{2}$ Present address: Taiwan Semiconductor Manufacturing Company, Taipei, Taiwan.

${ }^{3}$ Present address: Raytheon Company, Andover, MA, USA.
}

the ferroelectric charge into the semiconductor; therefore, a semiconductor with excellent chemical compatibility with oxides is required. Oxidation at the interface degrades the electrical properties of the heterostructure and makes the use of buffer layers [3] necessary. An oxide semiconductor eliminates this problem, thus offering a chemically stable structure. Zinc oxide [4-6], indium oxide [7], tin oxide [8] and lithium niobate [1,9] have been studied for this purpose previously. $\mathrm{ZnO}$ has also been used as a buffer layer for $(\mathrm{Pb}, \mathrm{Zr}) \mathrm{TiO}_{3}(\mathrm{PZT})$ capacitors [10] and as the active layer for ferroelectric gate thin film transistors [11]. While the integration of PZT/ZnO has been demonstrated in some device configurations, the electronic properties of these heterostructures are still not well understood. In this work we explore the coupling of polarization in PZT with mobile charge density in the $\mathrm{ZnO}$ semiconductor and compare with models based on the electrostatics of the material heterostructure. PZT/ZnO heterostructures are studied through capacitance-voltage $(C-V)$ and ac conductance measurements on metal/PZT/ZnO structures. In addition, the $\mathrm{PZT} / \mathrm{ZnO}$ capacitors are applied to a basic passive circuit to 
exploit the switchable polarization of the $\mathrm{PZT} / \mathrm{ZnO}$ structures to shift resonance frequency.

\section{Experiment}

We fabricated metal-insulator-semiconductor-metal capacitors to study the structural and electrical properties of PZT/ZnO heterostructures. Platinized silicon wafers from Radiant Technologies were used as the bottom electrode for the capacitor structures. We obtained PZT thin films using a sol-gel process starting from a suspension of $\left(\mathrm{Pb}_{0.52}, \mathrm{Zr}_{0.48}\right) \mathrm{TiO}_{3}$ nanocrystals purchased from Nippon FerroTechnology Corporation. The platinized silicon substrates were cleaned and annealed at $700{ }^{\circ} \mathrm{C}$ prior to sol-gel deposition. The PZT nanocrystal suspension was spin-coated on the substrates at $3000 \mathrm{rpm}$ for $30 \mathrm{~s}$. The samples were then dry baked at $115^{\circ} \mathrm{C}$ for $15 \mathrm{~min}$ and annealed at $700{ }^{\circ} \mathrm{C}$ for $2 \mathrm{~min}$ in oxygen and nitrogen through a rapid thermal annealing process. Repeating this process twice yielded ferroelectric films of thickness $400 \mathrm{~nm}$, as measured using optical reflectance.

We fabricated metal-PZT-metal capacitors as control structures to independently examine the properties of the PZT thin films. The top platinum contacts were achieved using electron beam evaporation and a shadow mask, with a contact area of $3.8 \times 10^{-4} \mathrm{~cm}^{2}$. We fabricated metalferroelectric-semiconductor-metal capacitors by depositing $\mathrm{ZnO}$ thin films on PZT using pulsed laser deposition (PLD). The deposition temperature was $300^{\circ} \mathrm{C}$ and the oxygen partial pressure was $30 \mathrm{mTorr}$. The laser pulse energies ranged from 250 to $300 \mathrm{~mJ}$, corresponding to fluence values of $0.75 \mathrm{~J} \mathrm{~cm}^{-2}$ to $1 \mathrm{~J} \mathrm{~cm}^{-2}$. $\mathrm{ZnO}$ film thicknesses varied between 300 and $450 \mathrm{~nm}$. ZnO thin films deposited on insulating sapphire substrates under similar deposition conditions typically yield an electron concentration of approximately $10^{18} \mathrm{~cm}^{-3}$ and electron mobility of approximately $10 \mathrm{~cm}^{2} \mathrm{~V}^{-1} \mathrm{~s}^{-1}$. Top ohmic contacts to the $\mathrm{ZnO}$ material were obtained by evaporation of a $\mathrm{Ti} / \mathrm{Al} / \mathrm{Au}$ metal stack through a shadow mask.

To evaluate the dielectric properties of the PZT thin films we carried out polarization-electric field $(P-E)$ measurements using a Radiant RT66A Ferroelectric Characterization System. We carried out these measurements on the control structures (metal-ferroelectric-metal), in order to ensure that our PZT thin films exhibited ferroelectric behaviour. We measured the capacitance-voltage $(C-V)$ characteristics of the $\mathrm{Pt} / \mathrm{PZT} / \mathrm{ZnO} / \mathrm{Pt}$ capacitors for a frequency range $5 \mathrm{kHz}-$ $1 \mathrm{MHz}$. The ac amplitude of the voltage signal was $10 \mathrm{mV}$ and the dc voltage sweep was done with a step size of $100 \mathrm{mV}$, at a rate of $100 \mathrm{~ms}$ per step.

\section{Capacitance-voltage and ac conductance measurements}

The $P-E$ results for $\mathrm{Pt} / \mathrm{PZT} / \mathrm{Pt}$ capacitors indicate ferroelectric behaviour for the sol-gel PZT material, as shown in figure 1 $(a)$. The Pt/PZT/Pt capacitors showed a remanent polarization of $30 \mu \mathrm{C} \mathrm{cm}^{-2}$ and a coercive field of $60 \mathrm{kV} \mathrm{cm}^{-1}$ corresponding to an applied bias of $2.5 \mathrm{~V}$. Capacitance-voltage $(C-V)$ characteristics of the $\mathrm{Pt} / \mathrm{PZT} / \mathrm{Pt}$ capacitors are shown in figure $1(b)$, demonstrating the characteristic 'butterfly' shape
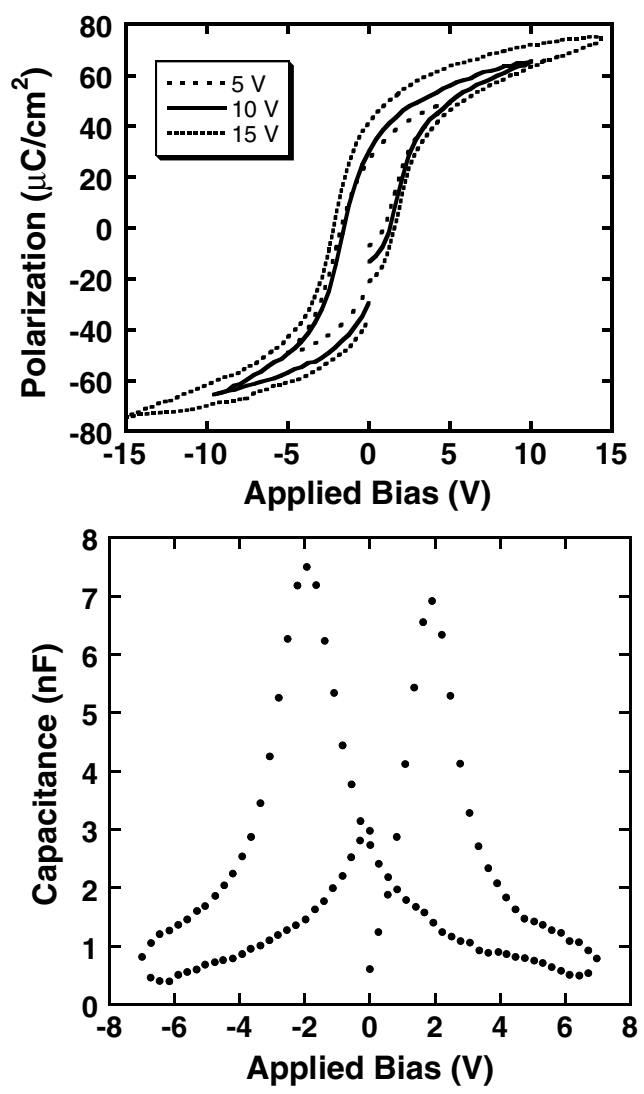

Figure 1. Ferroelectric behaviour of $\mathrm{Pt} / \mathrm{PZT} / \mathrm{Pt}$ thin film capacitors as shown by $(a)$ polarization-electric field and (b) capacitance-voltage measurements.

associated with ferroelectric thin films. The electric-field dependent dielectric constant of the PZT film ranged from $\varepsilon_{\mathrm{r}}=82 \varepsilon_{0}$ to $330 \varepsilon_{0}$. The peak capacitance value occurs at applied bias of $\pm 2.5 \mathrm{~V}$, corresponding to the coercive field.

We get an understanding of the coupling of ferroelectric polarization to the $\mathrm{ZnO}$ semiconductor through the variable frequency $C-V$ measurements. The results of this measurement are shown in figure 2. A hysteretic behaviour is clear in the $C-V$ data, with a memory window of approximately $4 \mathrm{~V}$. There is a capacitance peak at $2.5 \mathrm{~V}$ for a $C-V$ sweep in the forward direction. The $C-V$ characteristics of the $\mathrm{Pt} / \mathrm{PZT} / \mathrm{ZnO} / \mathrm{Pt}$ capacitors represent a superposition of the expected metal-insulator-semiconductor behaviour in conjunction with the switchable polarization and field-dependent dielectric constant of the PZT.

The shape of the $C-V$ characteristic shows that the capacitors can be operated in depletion and accumulation modes, but not in inversion. We do not see inversion in our devices due to the short minority carrier lifetime of holes in the n-type $\mathrm{ZnO}$ layer. The $C-V$ characteristics do not show significant frequency dependence for the measured range, with a variation in peak capacitance of less than $20 \%$. The hysteretic behaviour and peaked nature of the $C-V$ characteristics will be analysed in more detail in the following discussion section. AC conductance characteristics of the $\mathrm{Pt} / \mathrm{PZT} / \mathrm{ZnO} / \mathrm{Pt}$ capacitors at $1 \mathrm{MHz}$ (figure 3) demonstrate hysteresis consistent with $C-V$ 


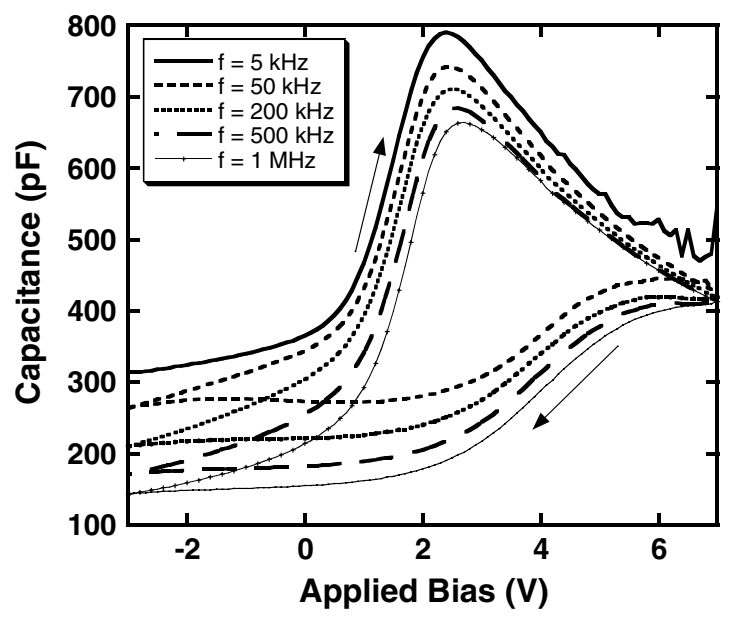

Figure 2. Capacitance-voltage characteristics of $\mathrm{Pt} / \mathrm{PZT} / \mathrm{ZnO}$ capacitors at variable frequency in forward and reverse sweep directions.

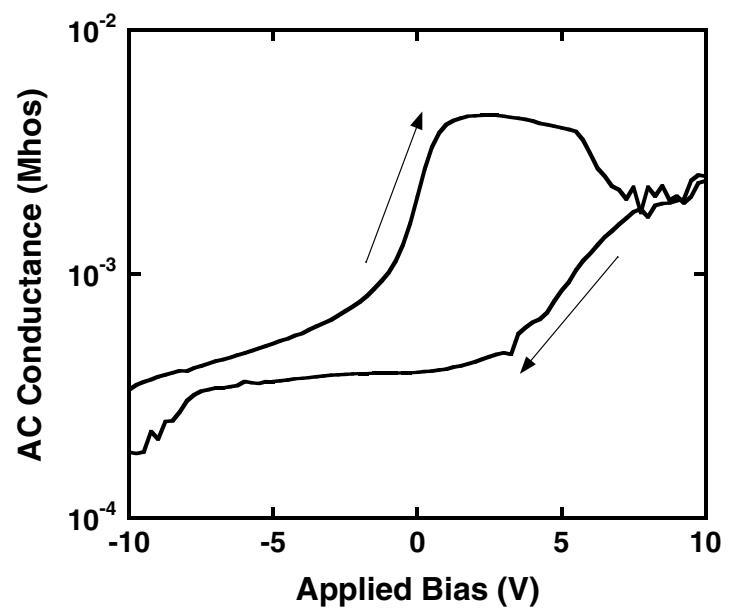

Figure 3. AC conductance of $\mathrm{Pt} / \mathrm{PZT} / \mathrm{ZnO}$ capacitors measured at $f=1 \mathrm{MHz}$ in forward and reverse sweep directions.

behaviour. The ac conductance demonstrates a significant change with bias and a memory window of $4 \mathrm{~V}$.

\section{Discussion}

The Pt/PZT/Pt and Pt/PZT/ZnO/Pt capacitors both exhibit a dc leakage current density with an ohmic (linear current versus voltage) behaviour for $\pm 10 \mathrm{~V}_{\mathrm{DC}}$. The leakage current density for Pt/PZT/Pt capacitors at $5 \mathrm{~V}_{\mathrm{DC}}$ applied bias is on the order of $10 \mu \mathrm{A} \mathrm{cm}^{-2}$. We believe the leakage current is primarily due to the polycrystalline characteristics of the PZT thin films, where conduction through the grain boundaries are the main source of current leakage through the insulator. The leakage current density for $\mathrm{Pt} / \mathrm{PZT} / \mathrm{ZnO} / \mathrm{Pt}$ capacitors was found to vary among differing samples, as well as varying among capacitors on a particular sample. In all cases, leakage current was significantly larger for the $\mathrm{Pt} / \mathrm{PZT} / \mathrm{ZnO} / \mathrm{Pt}$ capacitors, ranging from a factor of 10 to several orders of magnitude larger. The source of leakage current for the $\mathrm{Pt} / \mathrm{PZT} / \mathrm{ZnO} / \mathrm{Pt}$ structure will require further investigation. One possible source for leakage is related to the band lineup at the heterojunction interface between PZT and $\mathrm{ZnO}$, where a relatively small barrier to electron transport is expected. This is described in further detail in the following.

We believe the leakage current is primarily due to the polycrystalline characteristics of the PZT thin films, where conduction through the grain boundaries are the main source of current leakage through the insulator. In addition, leakage across the PZT/ZnO heterojunction may also contribute to current leakage. The band lineup at the heterojunction interface between PZT and $\mathrm{ZnO}$ is not well understood, where a relatively small barrier to electron transport may be expected, as described in further detail in the following. The bandgap energy for $\mathrm{ZnO}$ is known to be $3.3 \mathrm{eV}$ and the PZT bandgap included in the calculations was $3.7 \mathrm{eV}$. The electron affinity values used for PZT and $\mathrm{ZnO}$ were $3.5 \mathrm{eV}$ and $4.35 \mathrm{eV}$, respectively. Assuming a band lineup that follows the electron affinity, the structure has a type-II band lineup. The conduction band discontinuity is $0.85 \mathrm{eV}$, which also represents the barrier to electron transport from $\mathrm{ZnO}$ to PZT. It is well known that heterojunction band discontinuities can have large deviations from the electron affinity model and can also be strongly influenced by the defect structure at the interface. The interface characteristics of $\mathrm{ZnO} / \mathrm{PZT}$ heterojunctions are a subject for future study, where the electron affinity model will be used to provide a semi-quantitative analysis of the $C-V$ characteristics measured experimentally. We included the Pt electrode in the calculations with a work function of $5.3 \mathrm{eV}$. The band diagram of the resulting heterostructure for depletion and accumulation in the $\mathrm{ZnO}$ is shown in figures $4(a)$ and $(b)$. Calculated $C-V$ characteristics were obtained using the accumulation and depletion regions in metal-insulator-semiconductor structures [12]. We used a carrier concentration value of $1.8 \times$ $10^{18} \mathrm{~cm}^{-3}$ in the $\mathrm{ZnO}$, which we obtained through Hall-effect measurements of similar $\mathrm{ZnO}$ layers deposited on insulating substrates.

We calculated the $C-V$ characteristics using two models for the dielectric constant for PZT. The first model assumes a fixed dielectric constant for PZT and the second assumes a field-dependent dielectric constant. The field-dependent dielectric constant was modelled using a method developed by Chase et al, for BST thin films [13], where the capacitance is expressed as

$$
C(V)=\frac{C_{\max }}{\left[1+\left(\frac{2 V}{V_{2}}\right)^{2}\right]^{\gamma}},
$$

where $\gamma=0.417$. We used $300 \varepsilon_{0}$ for $C_{\max }$ and $3 \mathrm{~V}$ for $V_{2}$.

The resulting $C-V$ characteristics show good agreement with measured values, though there is still a deviation in the modelling of the peaked shape of the $C-V$, which may be due to a deviation in the modelling of the fielddependent permittivity in PZT. Figure 5 shows the measured and calculated $C-V$ characteristics of the ferroelectricsemiconductor capacitor at $1 \mathrm{MHz}$. Our calculation results suggest that the observed hysteresis would result from a density of sheet charges at the interface of magnitude $3 \times 10^{12} \mathrm{~cm}^{-2}$. This would correspond to a very small fraction of the polarization charge of the PZT capacitors, $1.3 \times 10^{14} \mathrm{~cm}^{-2}$. 

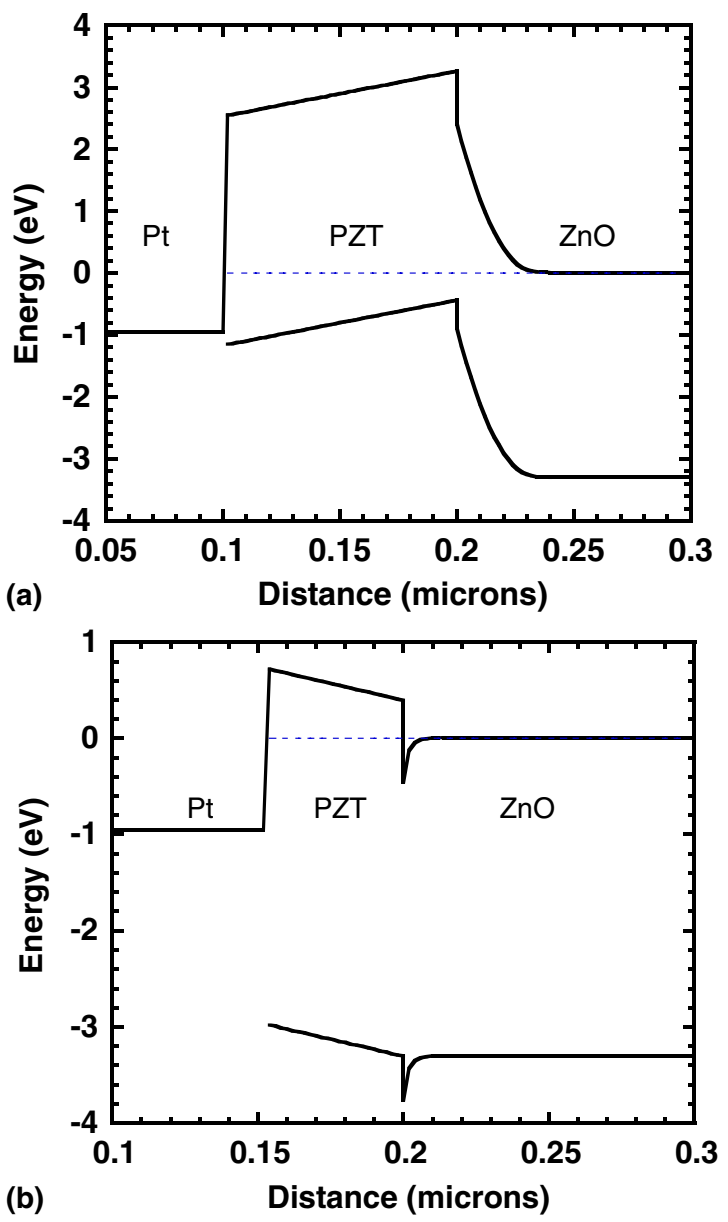

Figure 4. Energy band diagrams calculated for Pt/PZT/ZnO capacitors at $(a) V=0 \mathrm{~V}$ with PZT poled in the reverse direction depicting depletion and $(b) V=2 \mathrm{~V}$ with PZT poled in the forward direction depicting accumulation.

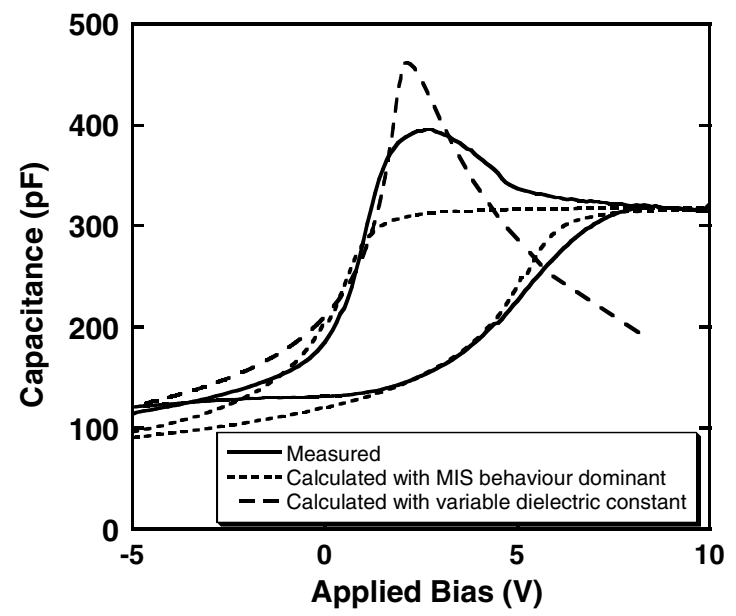

Figure 5. Comparison of calculated and measured $C-V$ behaviour at $1 \mathrm{MHz}$.

The variable frequency $C-V$ results (figure 2) show weak frequency dependence. This result leads us to believe that interface trap states are not significantly affecting the $C-V$ behaviour of the capacitors or that interface traps are at a shallow energy level with a fast response. The slope of

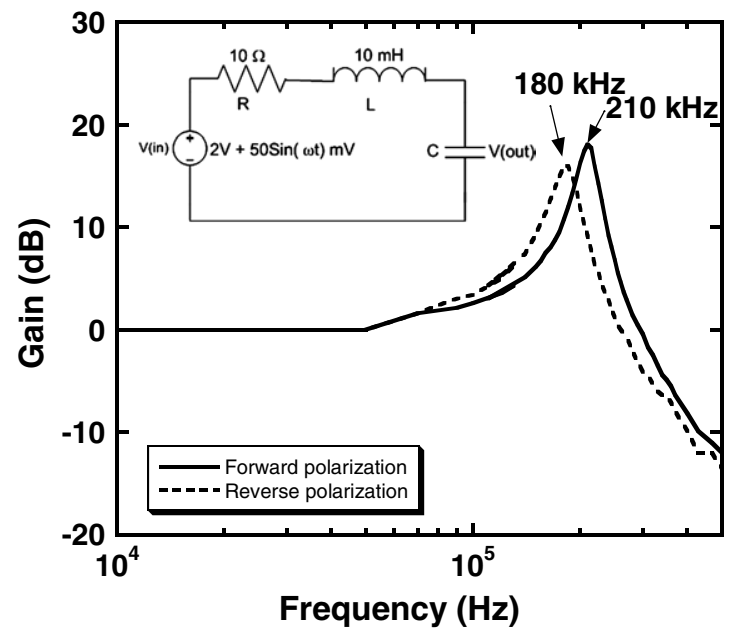

Figure 6. Frequency response of RLC circuit with PZT/ZnO capacitor in opposing polarization directions.

the $C-V$ characteristics also closely follows calculated $C-V$ characteristics. This suggests that energetically distributed traps at the interface are not strongly affecting $C-V$ behaviour, where such traps would be expected to result in a shallower slope in $C-V$. The hysteresis we observed in the ac conductance measurements is consistent with the $C-V$ results. Conductance is low with negative applied bias, because the device is in depletion mode. As the bias is swept in the positive direction, the device is put into accumulation mode and conductance increases by one order of magnitude. The device remains in depletion for a wider range of applied bias values when the sweep is in the positive-to-negative direction.

\section{PZT/ZnO capacitors in circuits}

We applied the $\mathrm{PZT} / \mathrm{ZnO}$ capacitors to a basic passive resonant circuit to further demonstrate hysteretic behaviour and potential utility of these structures. We used a series resistorinductor-capacitor (RLC) configuration for this purpose, as shown in figure 6 . The circuit values were chosen to provide a circuit with resonance frequency within the range where $C-V$ data were measured. The input consisted of a $2 \mathrm{~V}_{\mathrm{DC}}$ bias and a sinusoidal input signal with peak-to-peak amplitude of $100 \mathrm{mV}$. The PZT/ZnO capacitors were poled at $\pm 2 \mathrm{~V}_{\mathrm{DC}}$ prior to the measurement. The resulting frequency response of the circuit (figure 6) demonstrates peak resonance frequencies of 180 and $210 \mathrm{kHz}$ for opposing polarization directions, with a total resonant frequency shift of $30 \mathrm{kHz}$. The bandwidth of the resonance is approximately $30 \mathrm{kHz}$. The values of the PZT/ZnO capacitor corresponding to the $2 \mathrm{~V}_{\mathrm{DC}}$ operating point are 145 and $390 \mathrm{pF}$ for opposing polarization states. For these values the resonance frequencies are expected to be at 80 and $130 \mathrm{kHz}$, a shift of $50 \mathrm{kHz}$, which is similar to the measured value of $30 \mathrm{kHz}$. The expected bandwidth of the RLC circuit is $1 \mathrm{kHz}$, which is much smaller than the measured value of $30 \mathrm{kHz}$. We believe these differences are due to series resistance and parasitic capacitances corresponding to the test setup. 


\section{Conclusions}

We have presented electrical characteristics of $\mathrm{Pt} / \mathrm{PZT} / \mathrm{ZnO} / \mathrm{Pt}$ capacitors. The PZT thin films that we deposited through a sol-gel process showed ferroelectric hysteresis and a voltage tunable dielectric constant. $C-V$ measurements showed that the ferroelectric polarization charge of the PZT induces a charge density in the $\mathrm{ZnO}$. We observed a memory window of $2 \mathrm{~V}$ in the $C-V$ characteristics of the ferroelectricsemiconductor heterostructures. Variable frequency $C-V$ measurements showed that interface trap states had minimal effect on the capacitor performance. AC conductance measurements showed hysteretic behaviour consistent with our $C-V$ results. We also tested the ferroelectric-semiconductor capacitors in a simple RLC circuit. The test showed that the capacitors can be used to create a controlled shift in the resonant frequency of the circuit, making more sophisticated circuits applications possible.

\section{Acknowledgments}

This work is supported by the AFOSR under contract number FA9550-04-1-0390 and the National Science Foundation under Grant ECS-0238108.

\section{References}

[1] Rost T A, Lin H and Rabson T A 1991 Appl. Phys. Lett. 593654

[2] Shen B et al 2002 Mater. Res. Soc. Symp. Proc. 693 I11.41.1

[3] Fujisaki Y, Iseki K and Ishiwara H 2003 Mater. Res. Soc. Symp. Proc. $\mathbf{7 8 6} 297$

[4] Chen D Y, Murphy T E and Phillips J D 2005 Thin Solid Films 491301

[5] Lee S H, Ryu M K, Kim J P, Kim S R, Heo J H and Jang M S 2003 J. Korean Phys. Soc. 42 S1105

[6] Ashkenov N, Schubert M, Twerdowski E, Wenckstern T v, Mbenkum B N, Hochmuth H, Lorenz M, Grill W and Grundmann M 2005 Thin Solid Films 486153

[7] Evans J T, Suizu R I and Boyer L L 1997 Appl. Surf. Sci. 117/118 413

[8] Afanas'ev V P, Bulat D Y, Kaptelov E Y and Pronin I P 2004 Technol. Phys. Lett. 30518

[9] Kim K H 1998 Electron Devices Lett. 19204

[10] Wang X S, Wang Y J, Yin J and Liu Z G 2002 Scri. Mater. 46783

[11] Yoshimura T, Arai R, Masuko K, Ashida A and Fujimura N 2006 Japan. J. Appl. Phys. 45 L1266

[12] Casey H C (ed) 1999 Devices for Integrated Circuits: Silicon and III-V Compound Semiconductors (New York: Wiley)

[13] Chase D R, Chen L Y and York R A 2005 IEEE Trans. Microw. Theory $\mathbf{5 3} 3215$ 